\title{
Nanoparticle Structure by Coherent X-ray Diffraction
}

\author{
Ian Robinson \\ London Centre for Nanotechnology, University College London, London WC1H OAH, UK \\ Research Complex at Harwell, Didcot, Oxfordshire OX11 ODE, UK \\ email:i.robinson@ucl.ac.uk
}

This review examines the physical reasons why nanoparticles differ in structure from the bulk. Certain simple properties of nanoparticles are explained through these structural differences. A powerful method of measuring the three dimensional structure of nanoparticles, Coherent X-ray Diffraction, is introduced. A key experiment is described that uses CXD to study the redistribution of strains on the surface of a $A u$ nanocrystal. Some future perspectives are discussed in conclusion.

KEYWORDS: nanocrystal, equation of state, coherent X-ray diffraction, crystal structure, surface structure, strain, X-ray imaging, ...

\section{Physical properties of nanoparticles}

The basic physical characterisations of any solid material are encapsulated in its "equation of state", which provides the equilibrium structure and composition as a function of the most basic thermodynamic variables, temperature and pressure. A first approximations to extend this concept to nanomaterials is to add a third dimension to this picture, their size. We are therefore starting to see phase diagrams plotted (at constant pressure) as a function of temperature and size. New phases are being discovered at small size that have sufficiently different physical properties that they can be usefully exploited in suitable applications.

The simplest general assumption one can make to explain why nanoparticles are different from the bulk phase of the same material is that they contain more surface. They also show electron confinement effects which give rise to shifted spectral properties. ${ }^{1)}$ It is not a coincidence that much of the interest in the structure of nanoparticles comes from the field of surface science, where a large amount of information is available. There are "rules of thumb" in surface science that a surface displays certain liquid like properties, such as interatomic diffusion, at a given fraction of the bulk melting temperature, for example $0.7 T_{M}$. A general understanding of this phenomenon is that surfaces tend to have softer vibration modes than the bulk and so have thermal properties that operate at lower temperatures than the bulk. 
Because of their large surface component, nanoparticles are generally expected to melt at lower temperatures than the bulk. As discussed in this review, this is found experimentally for clusters ${ }^{2)}$ with the deviation from bulk behaviour commencing at a size of $100 \mathrm{~nm}$.

\subsection{Structure of nanoparticles}

The role of the surface can be used in a straightforward argument to explain the simplest measure of the structure, the lattice constant. Surface stresses, measured by a variety of methods, for metals are usually tensile and relatively large, in the range of $2 \mathrm{~N} / \mathrm{m} .{ }^{3,4)}$ These are of course strongly influenced by the details of any surface reconstruction and the adsorption of foreign species, hence whether or not the surface is cleaned to the high standards of Ultra-high Vacuum (UHV) conditions. If such a stress is present on the surface of a spherical particle of radius $R$, the surface stress $\sigma_{S}$ will give rise to a pressure difference $\Delta P$ between the inside and outside, according to the classical Gibbs-Thomson equation, otherwise known as the Young-Laplace equation, used to explain the stability of soap bubbles among many other applications:

$$
\Delta P=\frac{2 \sigma_{S}}{R} .
$$

This pressure difference then compresses the particle by an amount given by the compressibility or bulk modulus, $K$, of the material. In an isotropic material, the change, $\Delta a$, of the lattice constant, $a$, follows at one third the rate of the volume,

$$
\frac{\Delta a}{a}=\frac{2 \sigma_{S}}{3 K R}
$$

Equation 2 was used in the 1960's to interpret electron microscope images of nanoparticles in order to measure surface stresses. ${ }^{5)}$ More recently, it has been used to observe the change in lattice parameter of nanopowders by Mittemeijer et. al. ${ }^{6)}$ The samples were prepared as small nanocrystalline powders and progressively annealed to higher temperatures in a automated system. On each annealing cycle, the particle diameter $D=2 R$ is given by the width of its in situ X-ray powder diffraction, while the lattice constant is given by the peak position. Plots of $\frac{\Delta a}{a}$ it vs. $1 / D$ have a slope given by $\sigma_{S}$, according to equation 2 , as shown in Fig 1 . The experiment gave good results for $\mathrm{Cu}$ and Pd powders, showing $\sigma_{S} \simeq 2 \mathrm{~N} / \mathrm{m}$, but showed a discrepancy at very small size for Ni powders that was attributed to grain boundaries at the interparticle contacts. Since the grains touch each other, the (free) surface tension is no longer relevant and needs to be replaced by an interfacial tension. If the sputtered Ni grains are randomly oriented with respect to each other, the material will be full of grain boundaries which cannot be as closed-packed as the crystalline grains. The partial lack of filling of all 


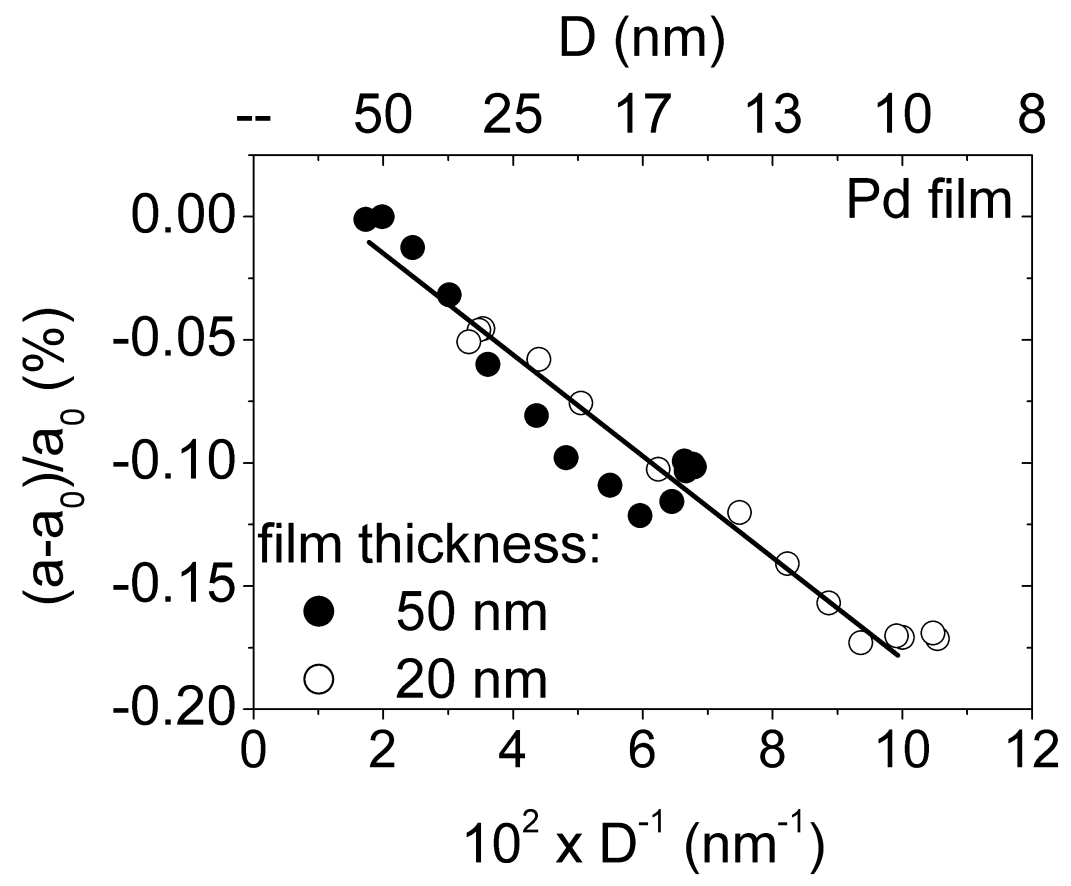

Fig. 1. Powder $X$-ray diffraction measurements of nanocrystalline powders of $P d$. Each point represents a different state of post annealing of the sample. The peak position is expressed as relative strain on the vertical axis. The reciprocal of the particle size, from the linewidth is horizontal. Reprinted with permission from "Nonmonotonic crystallite-size dependence of the lattice parameter of nanocrystalline nickel", J. Sheng, U. Welzel, and E. J. Mittemeijer, Appl. Phys. Lett. 97, 153109 (2010). Copyright 2010, American Institute of Physics.

atomic sites in these grain boundaries eventually results in a tensile force which causes the lattice constants to be expanded. This is what was observed for the smallest nanopowdered Ni samples measured by powder diffraction lineshape analysis. ${ }^{6}$

Individual gold nanocrystals were studied by Huang et. al. using coherent electron diffraction methods. ${ }^{78)}$ Careful model building, achieved on the atomic scale, found a distinct contraction of the outer layers of the crystal, as expected from equation 2. However when similar particles are thiolated, the outer layers are found to be expanded with respect to the bulk. ${ }^{9,10)}$ This is due to chemical invasion of the surface layers by the sulphur of the thiol used to coat the particles.

\subsection{Equation of State}

The simplest pictures of cohesion in solids involve some interatomic potential that is inherently anharmonic; the energy rises faster on compression than on expansion. Anharmonicity is responsible for the curvature of the compression response of a solid, usually called the "equation of state": the change of lattice constant with applied pressure follows a concave 


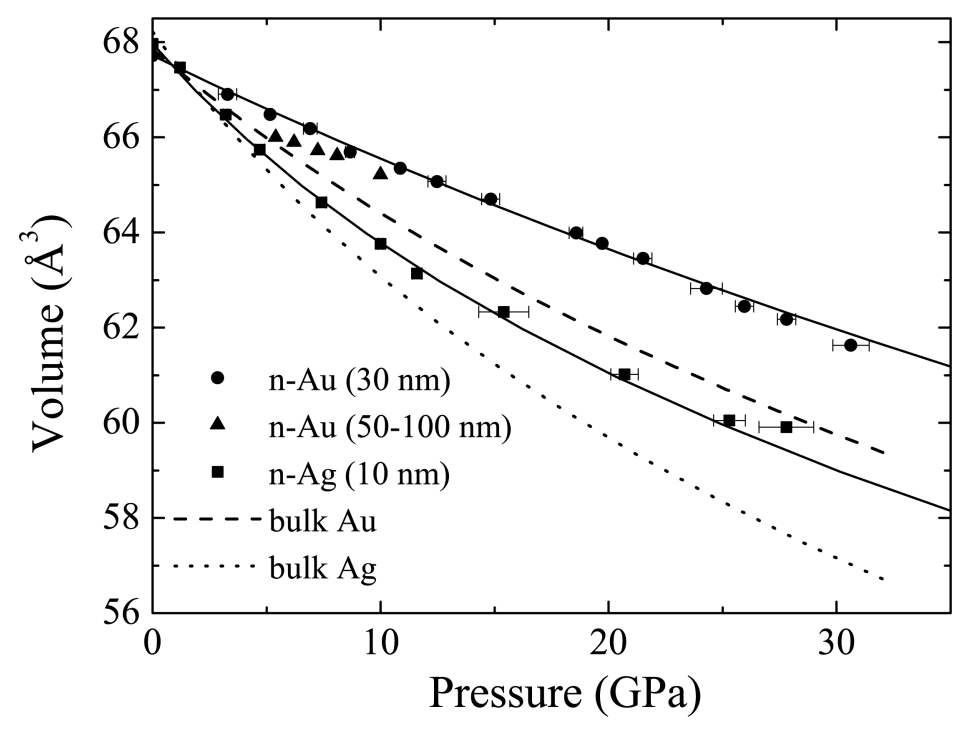

Fig. 2. Nano equation of state. Powder X-ray diffraction measurements of unit cell volume of nanocrystalline powders of $A u$ and $A g$ as a function of pressure measured in a Diamond Anvil Cell. Reprinted (abstract/excerpt/figure) with permission from "Unexpected High Stiffness of Ag and Au Nanoparticles", Q. F. Gu, G. Krauss, W. Steurer, F. Gramm, A. Cervellino, Phys. Rev. Lett. 100045502 (2008). Copyright (2008) by the American Physical Society.

shape whereby bigger pressure increments are required at higher pressures to produce the same change of lattice parameter. By extension of the arguments give above, it would be expected that nanoparticles, by virtue of their smaller lattice constant, would compress more slowly under added external pressure than their bulk counterparts. This expectation is encapsulated in the widely quoted maxim that "smaller is stronger".

The "nano equation of state" of $\mathrm{Ag}$ and Au nanopowders was measured by Gu et. al. using X-ray powder diffraction in a Diamond Anvil Cell. ${ }^{11)}$ The results of $\frac{\Delta a}{a} v s . P$, shown in Fig 2 , demonstrate a smaller slope and generally smaller pressure trend for the nanoparticles as compared with the bulk. For the sizes used, $10-100 \mathrm{~nm}$ diameter, they found approximately double the stiffness or bulk modulus, or half the amount of compressibility.

Predictions can therefore be made for the pressure response of larger nanoparticles. The crystal shapes, discussed below, comprise extended bulk-like regions and sharp corners. By virtue of the Gibbs-Thomson pressure, the sharp corner regions will be under compression 
and will show a stiffer response to pressure. Since the bulk-like regions of the larger nanoparticles will compress normally, a redistribution of strain would be expected upon application of pressure: the inhomogeneous distribution of strain would become further enhanced.

\subsection{Phase Diagrams}

Just as in classical phase diagrams of bulk materials showing phase transformations between different crystal structures as a function of temperature and pressure, it is expected that new phases would become stabilized as a function of the new thermodynamic variable, nanoparticle size. A good example of this behaviour is seen in the structures of semiconductor nanowires, often grown by the Vapor-Liquid-Solid (VLS) method. ${ }^{12,13)}$ InP is cubic (Zinc Blende) in the bulk but found to take the hexagonal Wurtzite structure in nanowires. ${ }^{14,15)}$ Other III-V semiconductors, such as GaP and GaAs, are apparently not as close to being unstable as nanowires in the cubic phase; nevertheless, they show a strong trend towards hexagonal stacking along their cubic 111 crystal directions by the introduction of frequent stacking faults. ${ }^{16)}$ One very interesting example is $\mathrm{Zn}$-doped InP nanowires which show periodic arrays of stacking faults intimately linked to the cross-sectional shape of the wires. ${ }^{14)}$ Along the wire, the shape evolves from a left-pointing triangle to a right-pointing triangle, then switches direction by the introduction of a stacking fault which reverses the trend in the next half-period. Many other materials are expected to show size-dependent changes of phase and are presumably waiting to be discovered. Among the transition metals, the stability of $C o$ and $\mathrm{Cu}$ lie close to the $\mathrm{HCP} / \mathrm{FCC}$ or BCC/FCC transition and can display the opposite phase in strained thin films. ${ }^{17}$ )

Nanocrystals have additional degrees of freedom from bulk phases in their choice of structure, because they are no longer bound by the constraints of long-range translational symmetry. Five-fold rotation axes are inconsistent with the long-range translational periodicity of a crystal lattice, so local 5-fold packing does not occur in crystals. Clusters and small nanoparticles can obtain lower energy, more stable, structures by employing 5-fold packing. For example, the central atom of an icosahedral cluster of 13 atoms is able to get closer to its neighbours than in a face-centred cubic (FCC) sphere-packing arrangement. Small clusters are sometimes found to display 'magic numbers' of preferred sizes, which correspond to closed icosahedral or decahedral shells. Famous examples are $A u_{13}$ and $A u_{55}$ clusters which are unusually stable and can be crystallised. ${ }^{9)}$

At slightly larger size, decahedral particle shapes occur containing a single 5-fold axis. ${ }^{18,19)}$ This results in elongated particles, which could in principle extend to 'nanorods' 
or even 'nanowires', since full translational symmetry can be maintained along the particle 5-fold rotation axis. A particularly elegant example of a decahedral crystal shape was discovered for the thiolated $A u_{104}(R S)_{44}$ cluster. ${ }^{10)}$ Here, a sufficiently monodispersed preparation of particles was synthesised that it could be crystallised. The crystal structure was solved to $1.1 \AA$ resolution by X-ray crystallography. The structure was found to be chiral with helical packing around its 5-fold axis; two enantiomorphs of opposite chirality appeared in the crystallographic unit cell. The cluster is decahedral in the core and contains a mixed Au$\mathrm{S}$ compound layer at the exterior, resulting in significantly enlarged $\mathrm{Au}-\mathrm{Au}$ spacings with the thiol sulphur atoms burying into the outer shell of the particles. ${ }^{10)}$ The chemistry of the sulphur atom is striking as it forms S-Au-S 'staples' with linear bonding of the central Au, three-fold coordination at the $\mathrm{S}$ with $90^{\circ}$ bonding of the two S-Au bonds and the thiol ' $\mathrm{R}$ ' ligand bisecting. The 'staples' were also been found to be stable theoretically ${ }^{20)}$ as well as experimentally in surface science studies of $\mathrm{S}$ on $\mathrm{Au},{ }^{21,22)}$ as well as in thiolated $A u_{25}$ clusters, which were found to contain a $A u_{13}\left(A u_{2}(R S)_{3}\right)_{6}$ core-shell chemical configuration. ${ }^{9)}$

\subsection{Structure of larger nanoparticles}

The surface free energy is the relevant quantity that determines the shapes of large crystals. By extension, when the effects of stress are included, this can be applied to nanocrystals. The surface energy is anisotropic because of the discreteness of the atomic structure at the surface. Close-packed facets are energetically favoured at low temperatures because their atoms have higher coordination number, while stepped regions become favourable at higher temperature due to the contribution of the configurational entropy of the steps to the free energy. The surface free energy as a function of crystal orientation displays a series of cusps, with the deepest ones corresponding to the lowest energy facets, for example 111 on face-centred cubic (FCC) crystals. $^{23)}$ The lowest energy shape of a crystal is therefore a compromise between the additional surface area needed to accommodate flat facets and the energy saved by the close packing. An elegant theory by $\mathrm{Wulff}^{24)}$ predicts that the equilibrium crystal shape is given by the Legendre transformation of the surface energy function of orientation. ${ }^{23,25)}$ The general result is an approximately spherical shape, upon which the steps are thermodynamically fluctuating at finite temperature, intercepted by flat facets. ${ }^{25,26)}$ As the temperature is increased, the area of the facets diminishes progressively. Facets can disappear altogether at a critical temperature called the roughening transition. ${ }^{27,28)}$

The structure of larger nanoparticles can be considered as an extension of this ideal, macroscopic bulk situation, once the strain due to the surface stress is introduced. While 
the surface free energy depends largely on temperature, the stress depends on the chemical state of the crystal surface and its environment, through contacts with neighbouring particles, substrates, solvents, surfactant layers etc. All finite crystals will have surface strains present due to these effects, but by the somewhat arbitrary definition of 'nanomaterials', nanocrystals are those in which the surface strain effects permeate the entire crystal and so significantly change the physical properties of the average material.

Surface free energy and surface stress are interrelated quantities, related through the Shuttleworth equation, ${ }^{29)}$ and measured in the same units of $N / m$ or $J / m^{2}{ }^{4)}$ The surface free energy is always positive and usually quite large because it accounts for the loss of cohesive energy (broken bonds) associated with the formation of a surface by breaking a bulk crystal. Surface stress can be positive or negative depending on the chemical environment, mentioned above, and state of reconstruction of the surface. Most metal surfaces have positive, 'tensile' surface stress due to the electronic contributions. ${ }^{4)}$ A simple explanation, often called "Smoluchowski smoothing", ${ }^{30)}$ is that a broken metal crystal will have rough boundaries of its Wigner-Seitz unit cells filled with electron density; when this redistributes to form a smoother boundary, the centre of mass of the electron density retracts inwards and Coulomb forces will cause a net inward contraction of the surface atoms leading to stress. This also leads to an inward relaxation of the surface metal ion core positions widely seen in the structure of metal surfaces. ${ }^{31)}$

The surface free energy determines the equilibrium crystal shape (ECS) of a nanocrystal, typically showing an alternating pattern of approximately spherical regions, with fluctuation steps, and atomically flat facets. The surface stress then acts on the spherical regions to produce a Gibbs-Thompson (GT) pressure, according to Equ(1), and the corresponding strain Equ(2). The relative fraction of facets and spherical surface within the ECS theory is independent of particle size, while the GT pressure and corresponding distortions of the crystal become bigger for smaller crystals. Rough estimates of the expected strains can be drawn from the numbers already used above. A 300 $\mathrm{nm}$ sized crystal might have corners of radius $R \simeq 30 \mathrm{~nm}$ from its ECS geometry. With a stress $\sigma_{S} \simeq 2 \mathrm{~N} / \mathrm{m}$ and a compressibility of $K \simeq 200 \mathrm{GPa}$, this will give rise to local strain variations of $0.1 \%$ between the corners and the facets. At the surface, such strains will correspond to lattice distortions of $0.03 \mathrm{~nm}$ or $10 \%$ of a lattice spacing.

The important conclusion is that metal nanocrystals should have a facetted shape and an inhomogeneous pattern of strain, with compressed segments under their spherical surface regions and relatively uncompressed segments under their flat facets. This is confirmed by 
experiments using coherent X-ray diffraction (CXD) methods described below. The image of Figure 3 shows the external surface of a free standing 300nm wide, 150nm tall Au nanocrystal, coloured according to its local strain, the measured component of the distortions of its lattice from an ideal crystal lattice. ${ }^{29,32)}$ The flattened shape of the crystal in Fig 3 can be understood because the time to reach equilibrium is prohibitively long and the true equilibrium crystal shape is never practically reached. Smaller nanocrystals would be expected to demonstrate these ECS effects more easily. The structure of this nanocrystal shows the distribution of nanoscale strains arising from the variation of local lattice constant around the surface. It is clear from the figure that the strain tends to follow the edges of the crystal shape, where the spherical regions are located. The distortions correspond to a quantitative phase variation of about 0.5 radians in good agreement with the expectations given above.

\subsection{Depression of melting point of nanoparticles}

The depression of melting point as a function of particle size in gold was measured by Buffat and Borel. ${ }^{2)}$ The melting point is seen to drop steadily with the inverse of the particle size eventually with quite substantial melting-point depressions, over $500 \mathrm{~K}$ for Au crystals below $2.5 \mathrm{~nm}$ in size. The effect was originally observed by Takagi in thin films under an electron microscope. ${ }^{33)}$ Similar melting point depression experiments were carried out using a microcalorimeter on Sn nanoparticles ${ }^{34)}$ and on oxide-coated Al nanoparticles, ${ }^{35)}$ all showing a similar trend. The effect is understood to be explained by the Gibbs Thomson (GT) pressure effect described above: the melting point depression, $\Delta T_{M}$, is proportional to the GT pressure $\Delta P$ due to the effect of surface stress in $\operatorname{Equ}(1)$, which increases as $1 / R$. Various models for the proportionality (and deviations from it) are in the literature, ${ }^{2,34,35)}$ but the simplest concept is the thermodynamic Clausius-Clapeyron equation describing the slope of the $P-T$ phase boundary in a phase diagram. This equivalent to saying that the vapour pressure of a small particle of radius $R$ is increased by an amount $\Delta P$ given by Equ(1), so it melts at a proportionately lower temperature.

\section{Coherent X-ray Diffraction}

A useful method of analysing the shapes and pattern of strains in nanocrystals is Coherent X-ray Diffraction (CXD). ${ }^{36,37)}$ The method relies on the use of highly coherent beams of Xrays generated by undulator sources of synchrotron radiation. When the beam is coherent across an entire crystal grain, its diffraction spots will be strongly modulated by interference between waves scattered by all its extremities. If the degree of coherence of the beam is 
insufficient, the visibility of these modulations would be reduced. If the coherence length of the source is matched to the size of the crystal, by the use of optics, the full coherent flux can be used, which can be in the range of $10^{8}$ to $10^{9}$ photons per second. For present day sources, this limits the minimum size of nanocrystals which can be studied to about $60 \mathrm{~nm}$ diameter.

The pattern of modulation appears as fringes surrounding each Bragg peak of diffraction from the crystal lattice. The simplest fringes are those which arise from interference between pairs of opposing facets on the crystal shape. Then the shape transform is a $\frac{\sin (2 \pi x / s)}{2 \pi x / s}$ ('sinc') function with a fringe spacing on the detector, $s$, given by the 'grating formula' $s=\frac{\lambda D}{d}$ where $\lambda$ is the wavelength, $d$ the distance between the facets (i.e. size of the sample), and $D$ is the detector distance. Spherical crystals give a circular fringe pattern given by a corresponding 3D Airy function. The facetted spherical shape discussed above will give a combination of both these patterns as an amplitude superposition which will display intermodulation of the patterns. An example of a coherent diffraction pattern is shown in Fig 3(c). This is the central frame of a rocking series measured around one of the $\{111\}$ Bragg peaks of the crystal shown in Fig 3(a) and (b). The stack of such frames, spanning the rocking curve, completely surrounds the reciprocal lattice point measured and so represents the complete diffraction pattern of the object in three dimensions (3D). ${ }^{36,37)}$

Such diffraction patterns are 'oversampled' with respect to the spatial Nyquist-Shannon sampling frequency. There are more pixels measured in each direction of the diffraction pattern than the half-period of the $\frac{\sin x}{x}$ ('sinc') function, so there are more measurements than the information needed to completely describe the finite-bandwidth object. Shortly after the publication of the Shannon sampling theorem, Sayre pointed out that such oversampled diffraction patterns contain enough information to solve the phase problem, so could be inverted to images. ${ }^{38)}$ However, it took from 1953 until 1999 for this to be demonstrated experimentally for X-rays by Miao. ${ }^{39)}$ Even though the X-ray detector cannot measure phase, the phase of the complex amplitude describing the Fourier transform of the nanoparticle shape, is embedded in the relative position of the fringes recorded. This missing phase can be recovered from the measured intensity data by a suitable algorithm, using the finite extent of the object (its 'support') as the only additional constraint. This mathematical phase retrieval problem has been extensively studied and found to have a unique solution in all but deliberately constructed "pathological" cases. ${ }^{40)}$ For CXD problems, the most important methodological breakthrough was the introduction of the "Hybrid Input-Output" (HIO) method by Fienup, ${ }^{41)}$ which was found to avoid stagnation of the algorithm. HIO is widely used today, but new methods such as those based on 'Compressed Sensing', ${ }^{42,43)}$ may provide an alternative route 
to a general solution method.

\subsection{Complex electron density}

An ideal nanocrystal, described by an external shape cutting a perfect lattice of atoms, would give rise to a CXD pattern that would be symmetric about each reciprocal lattice point. This follows from the inversion symmetry of the magnitude of the Fourier transform of a real object. Moreover, the CXD distribution surrounding every Bragg peak would be identical. Neither of these is true in practice because of the presence of strain within the nanocrystal. This can be used to image the strain: the inverse Fourier transform of the asymmetric diffraction pattern (once phased) is a complex density function. The magnitude of this function represents the electron density of the crystal, while the real-space phase image, $\phi(\mathbf{r})$, represents its distortion from an ideal lattice, mapped out as a function of position $\mathbf{r}$ within the crystal. $^{36,37)}$ This statement is fully quantitative and expressed by the relationship

$$
\phi(\mathbf{r})=\mathbf{Q} \cdot \mathbf{u}(\mathbf{r})
$$

where $\mathbf{Q}$ is the momentum transfer vector of the reciprocal lattice point measured and $\mathbf{u}(\mathbf{r})$ is the field of displacement vectors of the distortions from the ideal crystal lattice. The spatial derivative of the displacement field is the formal strain tensor. According to Equ(3), only one component of the displacement field is measured at a time: its projection onto the Q-vector. However if phase images from three or more independent Q-vectors are combined together, a full 3D reconstruction of $\mathbf{u}(\mathbf{r})$ can be made. This has been achieved for $\mathrm{ZnO}$ nanocrystals, for which full 3D images of each component of the full strain tensor have been published. ${ }^{44)}$

Visualisation of 3D vector fields is a generic challenge because these contain a wealth of information. Cross sections of individual components can be displayed as contour plots, but several sections are required to convey the full information. Given that the quantity measured is a real-space phase, a colour circle is a useful way to show it, but a full 3D map still requires multiple sections. For nanocrystals, the display method of Fig 3(a,b) has been found particularly useful. The strains tend to be mostly concentrated on the external surface and decay smoothly towards the interior; this is expected from the solution of the Poisson equation which governs the elastic response to stresses which arise on the surface. Fig 3(a,b) show isosurfaces, displayed at a single contour level, of the electron density to represent the shape of the crystal; the isosurface has then been coloured by the phase of the complex electron density, which then shows the surface variation of the imaged component of the displacement field. 

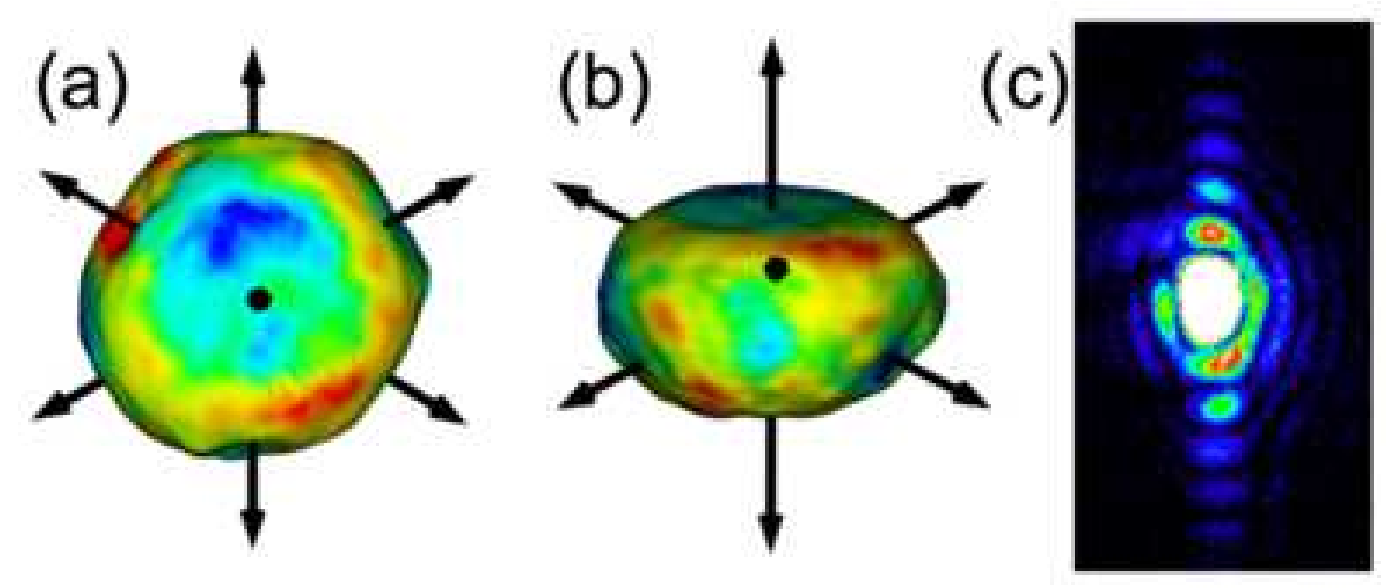

Fig. 3. (Color online) (a) Top view and (b) 111 side-facet view of a single nanocrystal of $A u$ measured by Coherent X-ray Diffraction (CXD). A single isosurface contour is shown, coloured according to the local strain, represented as real-space phase. The colour scale is from -0.75 radians (blue) to +0.75 radians (red). Eight arrows (length 250nm) denote the crystal 111 directions. (c) CXD pattern of a similar crystal measured on a Charge-coupled Device (CCD) at the centre of its rocking curve. ${ }^{29)}$

This Bragg CXD method is very sensitive. According to Equ(3), the phase changes by $2 \pi$ for every increment of the projection of $\mathbf{u}(\mathbf{r})$ by one lattice spacing. Thus the $10 \%$ spacing change envisioned in the introduction is sean as a clear signal of 0.6 radians. This is roughly the magnitude of the strains seen in Fig 3.

\subsection{In-situ thiolation of Au nanocrystal}

A recently published experiment ${ }^{29)}$ was carried out to investigate the stresses induced in an $A u$ nanocrystal by the formation of self-assembled monolayer (SAM) of thiol on its surface. ${ }^{45-48)}$ The crystals were made by dewetting a $20 \mathrm{~nm}$ thin film on a $S i$ wafer by annealing in a nitrogen atmosphere. ${ }^{37)}$ This method leads to crystals with a 'fibre' texture on the substrate, about $200 \mathrm{~nm}$ in diameter and spaced about $1 \mu \mathrm{m}$ apart. These crystals were transferred to the CXD diffractometer inside a nitrogen bag and measured at their off-specular $111 \mathrm{Bragg}$ peak. The image in Fig 3(a) is typical of the nanocrystals prepared in this way.

Immediately after measurement, a dose of propane thiol, $\mathrm{C}_{3} \mathrm{H}_{7} \mathrm{~S} \mathrm{H}$, was administered by forming a drop on ethanol solution on the tip of a syringe inside the nitrogen environment. The thiol evaporates and forms a SAM on the surface of the $A u$ nanocrystal. Without entering the hutch, a second CXD measurement was made of the in situ modified crystal. To visualise the small changes in real space, the Difference Fourier (DF) method ${ }^{49)}$ was used to create a 
3D map of the differences before and after formation of the SAM. This method is preferred to subtraction of images because it is insensitive to any errors that arise from the HIO phasing step. A second measurement, one hour later, found a similar but more pronounced set of changes. $^{29)}$

The changes in the structure of the $A u$ nanocrystal due to formation of the SAM are shown in Fig 4(a). The picture on the left shows a translucent image of the crystal, reoriented so that its $\mathbf{Q}$-vector is pointing up the page. An isosurface of the differences is superimposed, coloured according to the phase with yellow representing $\phi \simeq+\pi / 2$ and light blue representing $\phi \simeq-\pi / 2$. These represent equal and opposite relative displacements of the crystal in the forward and backwards direction of $\mathbf{Q}$, according to the DF formalism. ${ }^{49)}$ If the strains are radial on the surface of the crystal, it is expected that the biggest differences lie along $\mathbf{Q}$. The opposite sign means both ends of the crystal are expanded relative to the sides.

Fig 4(b) is a cross-section image of the vertical displacement simulated by Finite-element Analysis (FEA) to simulate this pattern of strain. In the FEA, a thin skin on the surface is explicitly stressed by changing its thermal expansion coefficient and applying a temperature change. In the example shown, a negative stress $\sigma_{S}=-1.5 \mathrm{~N} / \mathrm{m}$, was applied to the spherical facets of the model and no stress was applied to the facets, but the effect is virtually indistinguishable from other combinations with the same difference. ${ }^{29)}$ The simulated pattern, which strongly resembles the observation, shows a quantitative pattern of displacements in the picometre $(\mathrm{pm})$ range, which can be scaled to the measurement to obtain the stress difference. The measurement is not sensitive to absolute stress, which only causes a uniform pattern, but to the relative stresses applied. Using the known bulk modulus of gold the relative stress is determined to be $\Delta \sigma_{S}=4.5 \pm 2 \mathrm{~N} / \mathrm{m}$ rising to $\Delta \sigma_{S}=9.5 \pm 3 \mathrm{~N} / \mathrm{m}$ in the second measurement, one hour later. ${ }^{29)}$ The direction of the difference requires more positive stress on the facets than on the spherical regions, resulting in a relative inward displacement of the facets upon thiol adsorption.

Similar values of stress have been measured in micromechanical cantilever measurements $^{50,51)}$ of stresses generated by applying thiols to micron-thick $S i$ membranes coated on one side with $A u$. The specific $A u-S$ bonding causes stress on the coated surface of the cantilever, causing it to bend. ${ }^{52,53)}$ In experiments on $200 \mathrm{~nm}$-sized-grained films it was also found that the stress takes several hours to build. ${ }^{54,55)}$ Such cantilevers have important medical applications as sensors. ${ }^{56)}$ The fact that such large stress differences were detected in the CXD experiment indicates that the structure of the SAM must be very different between the flat and the curved surface regions of the nanocrystal. This is understandable when 


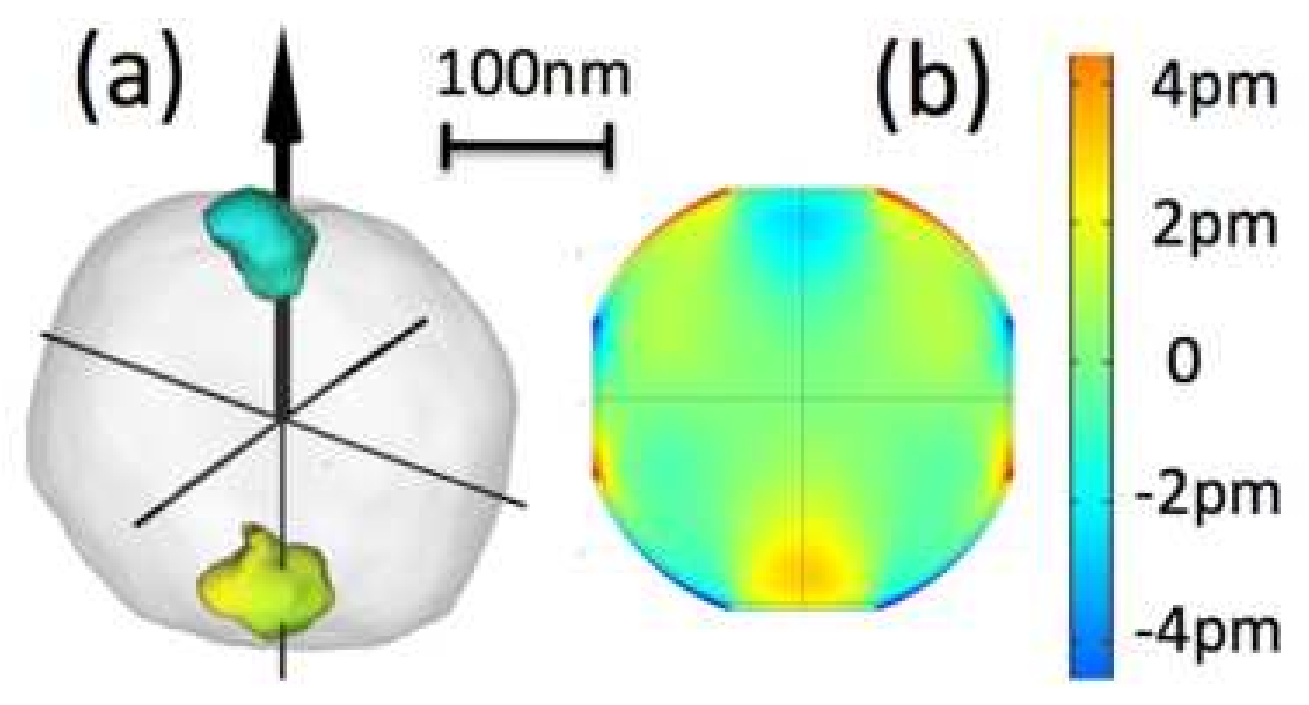

Fig. 4. (Color online) (a) Difference Fourier (DF) image of a $A u$ nanocrystal before and after thiol deposition. The isosurface is coloured by the phase of the differences found. (b) Finite-element Analysis (FEA) calculation of the vertical component of displacement arising from differential stress applied to the flat facets and curved regions. ${ }^{29)}$

it is considered that small radius thiolated nanoparticles form chemically intermixed layers of $S$ and $A u^{9,10)}$ by formation of 'staples' $;{ }^{20)}$ these regions have enlarged $A u-A u$ distances, while uncoated $A u$ nanoparticles are contracted. ${ }^{7}{ }^{18)}$ Surface science studies of SAMs formed on low-index surfaces, representative of the nanocrystal facets, are close-packed monolayers without intermixing. ${ }^{21,22)}$ So the conclusion is that, upon thiolation, the curved parts of the crystal form an intermixed thiol structure while the flat facets form more conventional SAMs.

\subsection{Future directions of CXD}

CXD is a generally useful tool for imaging the patterns of strain within nanocrystalline grains. Because it uses X-rays which penetrate many microns inside matter, a wide range of in situ applications can be envisaged to look at crystalline grains buried deep inside another material. UHV studies of the formation kinetics of nanocrystals by dewetting of thin films. Studies of alloy nanocrystals, where surface segregation effects are important, are likely to lead to 'core-shell' structures. It is expected that there will be not only core-shell structures formed by composition or choice of crystal structure, but also core-shell arrangements of strain itself, even in an otherwise single-phase material. Polycrystalline materials can be taken apart grain by grain, or assembled grain be grain to look at the strains expected whenever two 
grains start sintering together.

The spatial resolution attainable at present is in the region of $30 \mathrm{~nm}$, limited by counting statistics at the extremities of the diffraction patterns. This will get better as some low power $(1 / 3$ or $1 / 4)$ of both the incident flux (better sources) and exposure time (better stability). Current beamline stability, of optics with respect to the sample, limits the total experiment to about one hour, but this is likely to improve. Fortunately for the application of imaging strain fields, 30nm resolution is usually sufficient: strain fields are long range and slow varying. The length scale of the variation of strain follows that of the smallest feature in the structure, according to classical elasticity theory. For this reason, and because of the penetrating property, CXD will remain competitive with respect to transmission electron microscopy (TEM), which is capable of much higher resolution. It is interesting to note that a current trend in TEM is to analyse images in terms of 'geometric phase', ${ }^{57)}$ which is a lower-than-atomic resolution analogue of the phase described in $\mathrm{Equ}(3)$.

Some challenges lie ahead for CXD. Because of the need of a support surrounding the sample, constrained by the oversampling criterion, it has a limited field of view. Scanning versions of CXD based on the ideas of 'ptychography' ${ }^{58)}$ can solve this problem, but are still not fully developed for imaging strains. Another general limitation is the need to extend CXD to the case of large strains, with displacements bigger than a lattice spacing. In this case phase wrapping occurs in the measured quantity, the phase in Equ(3). This in turn results in diffraction patterns which are no longer "single centred", i.e. variations of the Airy function, but more like multi-peak "speckle" patterns instead. The HIO-based phasing algorithms are often found to fail in this case and will need to be improved.

\section{Acknowledgment}

Many colleagues contributed to the developments of the CXD methods described in this work. I particularly value the contributions of Moyu Watari, Rachel McKendry, Manuel Voegtli, Gabriel Aeppli, Yeong-Ah Soh, Xiaowen Shi, Gang Xiong, Xiaojing Huang, Jesse Clark, Ross Harder, Mark Pfeifer, Garth Williams and Ivan Vartanyants, who influenced the work and ideas presented here. The development of the methods described was supported by a FP7 grant 227711 "Nanosculpture" from the European Research Council. Beamtime for the experiment described was provided by the Advanced Photon Source, operated by the U.S. Department of Energy under Contract No. DE-AC02-06CH11357. The CXD instrument at Advanced Photon Source beamline 34ID-C was built with a National Science Foundation grant DMR-9724294. 


\section{References}

1) A. P. Alivisatos: Semiconductor clusters, nanocrystals, and quantum dots, Science 271 (1996) 933

2) P. Buffat, and J. Borel: Size Effect on Melting Temperature of Gold Particles, Physical Review A, 13 (1976) 2287

3) W. Haiss: Surface stress of clean and adsorbate-covered solids, Rep. Prog. Phys. 64 (2001) 591

4) H. Ibach: The role of surface stress in reconstruction, epitaxial growth and stabilization of mesoscopic structures, Surf. Sci. Rep. 29 (1997) 195263

5) H. J. Wasserman and J. S. Vermaak: Surf. Sci. 22 (1970) 164

6) J. Sheng, U. Welzel, and E. J. Mittemeijer: Nonmonotonic crystallite-size dependence of the lattice parameter of nanocrystalline nickel, Appl. Phys. Lett. 97 (2010) 153109

7) Huang, W. J., Sun, R., Tao, J., Menard, L. D., Nuzzo, R. G., and Zuo, J. M.: Coordinationdependent surface atomic contraction in nanocrystals revealed by coherent diffraction, Nature Mater. 7 (2008) 308313

8) I. K. Robinson: Giant molecules or tiny crystals?, Nature Materials, 7 (2008) 275

9) M. W. Heaven, A. Dass, P. S. White, K. M. Holt, and R. W. Murray: Crystal Structure of the Gold Nanoparticle $\left[\mathrm{N}\left(\mathrm{C}_{8} \mathrm{H}_{17}\right)_{4}\right]\left[\mathrm{Au}_{25}\left(\mathrm{SCH}_{2} \mathrm{CH}_{2} \mathrm{Ph}\right)_{18}\right]$, J. Am. Chem. Soc. 130 (2008) 3754

10) P. D. Jadzinsky, G. Calero, C. J. Ackerson, D. A. Bushnell, and R. D. Kornberg: Structure of a thiol monolayer-protected gold nanoparticle at 1.1 angstrom resolution Science 318 (2007) 430

11) Q. F. Gu, G. Krauss, W. Steurer, F. Gramm, and A. Cervellino: Unexpected High Stiffness of Ag and Au Nanoparticles, Phys. Rev. Lett. 100 (2008) 045502

12) A. Rastelli, M. Stoffel, J. Tersoff, G. S. Kar and O. G. Schmidt: Kinetic evolution and equilibrium morphology of strained islands, Phys. Rev. Lett. 95 (2005) 026103

13) Kodambaka, S., Tersoff, J., Reuter, M. C., and Ross, F.M.: Diameter-independent kinetics in the vapor-liquid-solid growth of Si nanowires, Phys. Rev. Lett. 96 (2006) 096105

14) Algra, R. E., Verheijen, M. A., Borgstrom, M. T., Feiner, L. F., Immink, G, van Enckevort, W. J. P., Vlieg, E, Bakkers, E. P. A. M.: Twinning superlattices in indium phosphide nanowires, Nature 456 (2008) 369 
15) Thelander, C, Agarwal, P, Brongersma, S, Eymery, J, Feiner, L. F., Forchel, A, Scheffler, M, Riess, W, Ohlsson, B. J., Gosele, U. and Samuelson, L: Nanowire-based onedimensional electronics, Materials Today 9 (2006) 28

16) V. Favre-Nicolin, F Mastropietro, J Eymery, D Camacho, Y M Niquet, B M Borg, M E Messing, L-E Wernersson, R E Algra, E P A M Bakkers, T H Metzger, R Harder and I K Robinson: Analysis of strain and stacking faults in single nanowires using Bragg coherent diffraction imaging, New Journal of Physics 12 (2010) 035013

17) B. M. Ocko, I. K. Robinson, M. Weinert, R. J. Randler and D. M. Kolb: Thickness-Induced Buckling of bcc Copper Films, Physical Review Letters 83 (1999) 780

18) L. D. Marks: Rep. Prog. Phys. 57 (1994) 603

19) P. M. Ajayan, and L. D. Marks: Phys. Rev. Lett. 60 (1998) 585587

20) D. Jiang, M. L. Tiago, W. Luo, and S. Dai: The Staple Motif: A Key to Stability of Thiolate-Protected Gold Nanoclusters, J. Am. Chem. Soc., 130 (2008) 2777

21) P. Maksymovych, O. Voznyy, D. B. Dougherty, D. C. Sorescu, and J. T. Yates: Gold adatom as a key structural component in self-assembled monolayers of organosulfur molecules on Au(111), Progress in Surface Science 85 (2010) 206

22) Miao Yu, N. Bovet, C. J. Satterley, S. Bengio, K. Lovelock, P. K. Milligan, R. G. Jones, D. P. Woodruff, and V. Dhanak: True Nature of an Archetypal Self-Assembly System: Mobile Au-Thiolate Species on Au(111), Phys. Rev. Lett 97 (2006) 166102

23) M. Wortis: in Chemistry and Physics of Solid Surfaces VII Springer Series in Surface Science 10, ed. R. Vanselow and R. Howe, (1988) 367

24) G. Wulff: Z. Krist. 34 (1901) 449

25) J. C. Heyraud and J. J. Metois: Equilibrium Shape and Temperature: Lead on Graphite, Surface Science 128 (1983) 334

26) L. Kuipers, M. S. Hoogeman, and J. W. M. Frenken: Step dynamics on Au(110) studied with a high-temperature, high-speed scanning tunneling microscope, Phys. Rev. Lett. 71 (1993) 3517

27) J. W. M. Frenken and J. F. van der Veen: Phys. Rev. Lett. 54 (1985) 134

28) Avron, J. E., van Beijeren, H., Schulman, L. S. and Zia, R. K. P.: Roughening transition, surface tension and equilibrium droplet shapes in a two-dimensional Ising system, Journal of Physics A: Mathematical and General, 15 (1982) L81 
29) M. Watari, R. McKendry, M. Voegtli, G. Aeppli, Y-A. Soh, X. Shi, G. Xiong, X. Huang, R. Harder and I. Robinson: Differential stress induced by thiol adsorption on facetted nanocrystals, Nature Materials 10 (2011) 862

30) R. Smoluchowski: Anisotropy of the Electronic Work Function of Metals, Phys. Rev. 60 (1941) 661

31) M. Finnis and V. Heine: Theory of Lattice Contraction at Al Surfaces, J. Phys. F - Metal Physics 4 (1974) L37

32) G. J. Williams, I. A. Vartaniants and I. K. Robinson: Orientation variation of surface strain, R. Harder, M. A. Pfeifer, Physical Review B 76 (2007) 115425

33) M. Takagi: Electron-Diffraction Study of Liquid-Solid Transition of Thin Metal Films, J. Phys. Soc. Jpn. 9 (1954) 359

34) S. L. Lai, J. Y. Guo, V. Petrova, G. Ramanath, and L. H. Allen: Size-Dependent Melting Properties of Small Tin Particles: Nanocalorimetric Measurements, Phys. Rev. Lett. 77 (1996) 99102

35) Sun J. and Simon S. L.: The melting behavior of aluminum nanoparticles, Thermochimica Acta 463 (2007) 32

36) I. K. Robinson and R. Harder: Coherent Diffraction Imaging of Strains on the Nanoscale, Nature Materials 8 (2009) 291

37) M. A. Pfeifer, G. J. Williams, I. A. Vartanyants, R. Harder, and I. K. Robinson: Threedimensional Mapping of a Deformation Field inside a Nanocrystal, Nature 442 (2006) 63

38) D. Sayre: Some Implications of a Theorem due to Shannon, Acta Crystallographica 5 (1953) 843

39) Miao, J., Charalambous, P., Kirz, J. and Sayre, D., Nature, 400 (1999) 342344

40) R. H. T. Bates: Fourier phase problems are uniquely solvable in more than one dimension, Optik, 61 (1981) 247

41) J. R. Fienup: Phase retrieval algorithms: a comparison, Appl. Opt. 21 (1982) 2758

42) M. C. Newton, R. Harder, Xiaojing Huang, Gang Xiong and I. K. Robinson: Phase Retrieval of Diffraction from Highly Strained Crystals, Physical Review B 82 (2010) 165436

43) Saghi, Z., Holland, D.J., Leary, R., Falqui, A., Bertoni, G., Sederman, A.J., Gladden, L.F., and Midgley, P.A.: Three-dimensional morphology of iron oxide nanoparticles with reactive concave surfaces. A compressed sensing-electron tomography (CS-ET) approach, Nanoletters 11 (2011) 4666 
44) M. C. Newton, S. J. Leake, R. Harder and I. K. Robinson: Three-dimensional imaging of strain in a single $\mathrm{ZnO}$ nanorod, Nature Materials 9 (2010) 120

45) R. G. Nuzzo and D. L. Allara: Adsorption of bifunctional organic disulfides on gold surfaces, J. Am. Chem. Soc. 105 (1983) 4481

46) C. D. Bain, E. B. Troughton, Y. T. Tao, J. Evall, G. M. Whitesides and R. G. Nuzzo: Formation of monolayer films by the spontaneous assembly of organic thiols from solution onto gold, J. Am. Chem. Soc. 111 (1989) 321

47) J. C. Love, L. A. Estroff, J. K. Kriebel, R. G. Nuzzo and G M. Whitesides: Self-Assembled Monolayers of Thiolates on Metals as a Form of Nanotechnology, Chem. Rev. 105 (2005) 1103

48) M. Kim, J. N. Hohman, Y. Cao, K. N. Houk, H. Ma, A. K.-Y. Jen and P. S. Weiss: Creating Favorable Geometries for Directing Organic Photoreactions in Alkanethiolate Monolayers, Science 331 (2011) 1312

49) R. Henderson and J. K. Moffat: The difference Fourier technique in protein crystallography: errors and their treatment, Acta Cryst. B27 (1971) 1414

50) R. A. McKendry, J. Zhang, Y. Arntz, T. Strunz, M. Hegner, H-P. Lang, M.K. Baller, U. Certa, H-J. Guntherodt, and C. Gerber: Multiple label-free biodetection and quantitative DNA binding assays on a nanomechanical cantilever array, Proc. Natl. Acad Sci. U.S.A. 99 (2002) 9783

51) M. Watari, J. Galbraith, H. P. Lang, M. Sousa, M. Hegner, C. Gerber, M. A. Horton and R. A. McKendry: Investigating the molecular mechanisms of in-plane mechanochemistry on cantilever arrays, J. Am. Chem. Soc. 129 (2007) 601

52) J. Fritz, M.K. Baller, H.P. Lang, H. Rothuizen, P. Vettiger, E. Meyer, H.-J. Guntherodt, Ch. Gerber and J.K. Gimzewski: Translating Biomolecular Recognition into Nanomechanics, Science 288 (2000) 316

53) R. Berger, E. Delamarche, H-P. Lang, C. Gerber, J. K. Gimzewski, E. Meyer, H-J. Guentherodt: Surface Stress in the Self-Assembly of Alkanethiols on Gold, Science 276 (1997) 2021

54) M. Godin, P. J. Williams, V. Tabard-Cossa, O. Laroche, L. Y. Beaulieu, R. B. Lennox and P. Grutter: Surface Stress, Kinetics, and Structure of Alkanethiol Self-Assembled Monolayers, Langmuir 20 (2004) 7090 
55) M. Godin, V. Tabard-Cossa, Y. Miyahara, T. Monga, P. J. Williams, L. Y. Beaulieu, R. B. Lennox and P. Grutter: Cantilever-based sensing: the origin of surface stress and optimization strategies, Nanotechnology 21 (2010) 075501

56) J. W. Ndieyira, M. Watari, A. D. Barrera, D.Zhou, M. Voegtli, M. Batchelor, M. A. Cooper, T. Strunz, M. A. Horton, C. Abell, T. Rayment, G. Aeppli and R. A. McKendry: Nanomechanical detection of antibiotic mucopeptide binding in a model for superbug drug resistance, Nature Nanotechnology 3 (2008) 691

57) Hytch, M J, Houdellier, F, Hue, F, and Snoeck, E: Dark-field electron holography for the measurement of geometric phase, Ultramicroscopy 111 (2011) 1328

58) Rodenburg, J. M., Hurst, A. C., Cullis, A. G., Dobson, B. R., Pfeiffer, F., Bunk, O., David, C., Jefimovs, K., and Johnson, I.: Hard-X-ray lensless imaging of extended objects, Phys. Rev. Lett. 98 (2007) 034801 